\title{
DENSIDAD POBLACIONAL Y CARACTERIZACIÓN DE HÁBITAT DEL VENADO COLA BLANCA EN UN BOSQUE TEMPLADO DE OAXACA, MÉXICO
}

\author{
EVELYN PIÑA ${ }^{1}$ \& IRMA TREJO \\ Instituto de Geografía, UNAM. Circuito Exterior s/n, Ciudad Universitaria, C. P. 04510, México, D. F. \\ ${ }^{1}<$ mustela_nigripes@yahoo.com.mx $>$
}

Piña, E. \& Trejo, I. 2014. Densidad poblacional y caracterización de hábitat del venado cola blanca en un bosque templado de Oaxaca, México. Acta Zoológica Mexicana (n.s.), 30(1): 114-134.

RESUMEN. Se evaluó la densidad poblacional y se caracterizó el hábitat del venado cola blanca Odocoileus virginianus en un bosque templado de Santa María Yavesía, Sierra Norte de Oaxaca. Se realizaron dos muestreos temporales en 13 sitios pertenecientes a cinco asociaciones vegetales con el método de conteos de grupos fecales. Se calculó la densidad poblacional con el modelo de Eberhardt \& Van Etten (1956) usando dos tasas de defecación. Se registró la presencia de venados sólo en el bosque de coníferas y el bosque de pino-encino cerrado. Las densidades promedio encontradas entre marzo a julio de 2008 fueron de $1.30 \pm 3.04$ individuos $/ \mathrm{km}^{2}$ y de $0.49 \pm 1.14$ individuos $/ \mathrm{km}^{2}$. Se midieron 13 variables del hábitat del venado cola blanca (vegetación y atributos físicos). Los sitios en el bosque de coníferas están a mayor altitud, son más fríos y húmedos, tienen más árboles grandes y un estrato arbustivo abierto. Las densidades poblacionales encontradas en el presente estudio fueron bajas, comparadas con las encontradas en otros estudios realizados con el método de conteo de grupos fecales en el país. Se proponen recomendaciones de manejo para los venados cola blanca de Santa María Yavesía.

Palabras clave: Densidad poblacional, caracterización de hábitat, Santa María Yavesía, Oaxaca, Artiodactyla, Odocoileus virginianus

Piña, E. \& Trejo, I. 2014. Population density and habitat characterization of the white-tailed deer in a temperate forest of Oaxaca, Mexico. Acta Zoológica Mexicana (n.s.), 30(1): 114-134.

ABSTRACT. The population density and the habitat characterization of the white-tailed deer Odocoileus virginianus were assessed in a temperate forest near Santa María Yavesía, Sierra Norte of Oaxaca. Two sample surveys were carried out at 13 sites belonging to five plant associations using the pellet group count method. The population density was calculated with the Eberhardt \& Van Etten (1959) model using two defecation rates. The presence of deer was only registered in the coniferous forest and the closed pine-oak forest. The average densities found between March and July 2008 were $1.30 \pm 3.04$ individuals $/ \mathrm{km}^{2}$ and $0.49 \pm 1.14$ individuals $/ \mathrm{km}^{2}$. Thirteen variables (vegetation and physical attributes) of the white-tailed deer habitat were measured. The sites of the coniferous forest are found at higher altitudes, are colder, have more humidity, have a higher number or larger trees and an open tree and shrub strata. The population densities found in this study were low compared with densities found in other

Recibido: 07/05/2013; aceptado: 24/10/2013. 
studies carried out in Mexico with the pellet group count method. Some management recommendations are proposed for the white-tailed deer of Santa María Yavesía.

Key words: Population density, habitat characterization, Santa María Yavesía, Oaxaca, Artiodactyla, Odocoileus virginianus

\section{INTRODUCCIÓN}

La conservación y el manejo de los recursos naturales deben involucrar su supervivencia, el bienestar humano y la participación de las poblaciones locales (De Fries et al. 2005). Añadirle valor económico a las especies genera incentivos socioeconómicos para su conservación. En particular, el aprovechamiento cinegético puede representar una alternativa productiva sustentable que ofrezca los incentivos económicos necesarios para detener y modificar las prácticas de manejo inadecuadas, lo que derivará en la conservación de las especies, así como de los hábitats en los que se encuentran (Argüeyes 2008; Villarreal 2006).

El venado cola blanca (Odocoileus virginianus) es la especie usada por excelencia para actividades cinegéticas, por lo que tiene una gran importancia económica, hecho que realza la necesidad de establecer el estatus actual de sus poblaciones, así como su hábitat, sobre todo cuando se trata de poblaciones silvestres que se pretenden manejar de manera sustentable (Dasmann 1971; Galindo-Leal 1993; Leopold 1977; McCullough 1987; Wilson et al. 1996). La posibilidad legal de obtener beneficios económicos derivados de su aprovechamiento influye en el cambio de actitud para cuidar y recuperar el hábitat y sus poblaciones locales en algunas comunidades rurales del país (Ortiz-Martínez et al. 2005). Es por ello que es urgente plantear alternativas de manejo para su conservación y aprovechamiento adecuado (Mandujano 2004).

El venado cola blanca tiene una importancia ecológica como herbívoro y presa, formando parte de redes tróficas, comunidades y ecosistemas diversos. Es un importante regulador de poblaciones vegetales, actuando como dispersor de semillas y ejerce un efecto en la estructura de la vegetación de los bosques con el ramoneo que realiza. Actúa como un factor limitante fundamental para poblaciones de grandes depredadores, siendo presa natural del jaguar, puma, lobo gris mexicano y oso gris mexicano, entre otros (Galindo-Leal \& Weber 1998; Valenzuela 1991; Waller \& Alverson 1997). A medida que disminuye su abundancia, los depredadores pueden cambiar su dieta, consumiendo ganado doméstico, lo cual ocasiona conflictos humano-vida silvestre que pueden poner en riesgo la supervivencia de especies en peligro de extinción (Foster et al. 2009; Núñez et al. 2002; Polisar 2002; Sáenz \& Carrillo 2002).

Puede encontrarse en prácticamente todos los tipos de hábitat del país, siempre y cuando éstos le proporcionen suficiente refugio y alimento. No es común en las zonas más secas y abiertas de matorral xerófilo ni en las partes más densas y húmedas del bosque tropical perennifolio (Aranda 2000). Se distribuye desde el sur de Canadá, 
prácticamente desde cerca del límite de crecimiento de los árboles, hasta Brasil y Perú (15 de latitud S; Halls 1984; Smith 1991). En México, es el cérvido con la distribución más amplia y puede encontrarse en todo el territorio nacional, con excepción de Baja California (Leopold 1977). En la región de la Sierra Norte de Oaxaca habita la subespecie Odocoileus virginianus oaxacensis, considerada endémica de la región (Hall 1981) y con un área de distribución aproximada de $30,000 \mathrm{~km}^{2}$ (Villarreal 2006). El conocimiento del estado actual de sus poblaciones es crítico, ya que es una de las subespecies menos estudiadas del país (Briones-Salas \& García 2005; Mandujano 2004; Ortiz-Martínez et al. 2005), es aprovechada (cacería de subsistencia) por grupos indígenas y campesinos en toda su área de distribución (González \& Briones-Salas 2000; Mandujano 2004) e incrementos sustanciales en sus poblaciones, así como recuperaciones en su hábitat podrían lograrse si su caza fuera regulada (Galindo-Leal \& Weber 1998; Leopold 1977; Mandujano \& Rico-Gray 1991; Martínez et al. 1997; Ortiz-Martínez et al. 2005) y porque podría ser una opción de impulso para la economía de las comunidades que desearan explorar alternativas de explotación no extractivas, como el ecoturismo (Galindo-Leal \& Weber 1998). Éste es el caso de la comunidad indígena de Santa María Yavesía, en donde el venado cola blanca presenta una oportunidad para diversificar el aprovechamiento de sus recursos naturales.

En este trabajo se estimó la densidad poblacional del venado cola blanca en cinco asociaciones vegetales pertenecientes a un bosque templado, se caracterizó su hábitat con base en 13 variables (vegetación y físicas) y se evaluó si las diferencias en la calidad de hábitat influyen en la presencia del venado cola blanca.

\section{MATERIAL Y MÉTODOS}

Área de estudio. Santa María Yavesía se encuentra ubicada en el Distrito de Ixtlán, dentro de la Sierra Norte y forma parte de la Región Centro-Norte del estado de Oaxaca. Se localiza entre las coordenadas $17^{\circ} 08^{\prime} 30^{\prime \prime}$ - $17^{\circ} 15^{\prime} 45^{\prime \prime} \mathrm{N}$ y $96^{\circ} 21^{\prime} 15^{\prime}$ ' - 96 $27^{\circ} 45^{\prime}$ ' W y cuenta con una extensión territorial de 6,455 ha (Fig. 1). Al norte colinda con el municipio de Santiago Xiacui, al este con Santiago Laxopa, al oeste con San Miguel Amatlán y al sur con Villa Díaz Ordaz. Comprende un intervalo altitudinal que va de los 1,900 a los 3,280 m s. n. m. (Aguilar 2007; Jiménez 2004). En la parte alta se presenta un clima semifrío subhúmedo $\left(\mathrm{Cb}^{\prime}(\mathrm{w} 2)(\mathrm{w}) \mathrm{ig}\right)$ con precipitación de $1,123 \mathrm{~mm}$ y temperatura media anual de $9.9^{\circ} \mathrm{C}$, un régimen de lluvias en un verano fresco y largo, mientras que en la parte baja el clima es templado subhúmedo $\left(\mathrm{Cb}(\mathrm{w} 2)(\mathrm{w})\right.$ ig), con precipitación de $1,030 \mathrm{~mm}$, temperatura media anual de $16.7^{\circ} \mathrm{C}$ y un régimen de lluvias en un verano fresco y largo. En la parte alta $(2,900 \mathrm{~m} \mathrm{~s} . \mathrm{n}$. m.) existen bosques de coníferas, que en algunas zonas se entremezclan conformando asociaciones. Hacia la parte media (alrededor de los 2,500 m s. n. m.) dominan bosques mixtos que presentan diferencias en abundancias de especies de Pinus, con un 


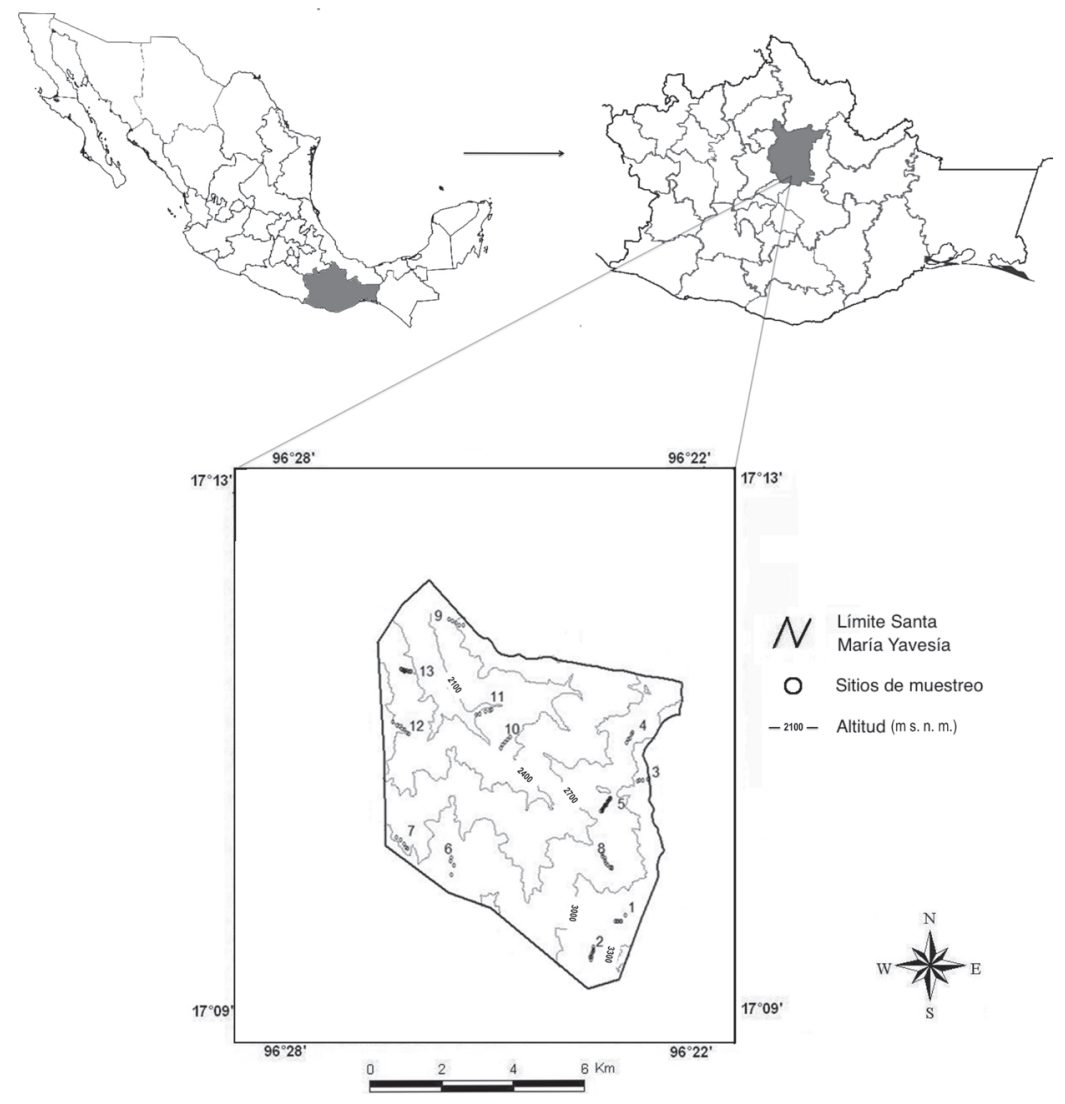

Figura 1. Mapa de 13 sitios de muestreo para el venado cola blanca en Santa María Yavesía.

sotobosque constituido principalmente por especies de Quercus. Finalmente, alrededor de los 2,000 m s. n. m. existen encinares caducifolios (Benítez 2006).

La colecta de plantas, el uso de leña y madera y la caza de animales son parte de las actividades de los pobladores, siendo esta última una práctica regular, cazándose principalmente ardillas (Glaucomys volans y Sciurus aureogaster), mapaches (Procyon lotor), armadillos (Dasypus novemcinctus), comadrejas (Mustela frenata), zorras (Urocyon cinereoargenteus) y venados cola blanca (Ambriz 2003; Ramos En proceso).

Densidad poblacional. Se seleccionaron 13 sitios (Fig. 1) pertenecientes a cinco asociaciones vegetales: bosque de coníferas (cuatro sitios), bosque de pino-encino abierto (tres sitios), bosque de pino-encino cerrado (cuatro sitios), bosque de encinopino (un sitio) y encinar caducifolio (un sitio). Se realizaron dos muestreos (marzo 2008, junio-julio 2008; Piña 2010). 
Para estimar la densidad poblacional del venado cola blanca se utilizó el método de conteos de grupos fecales, por ser el método más consolidado para la estimación de la abundancia de cérvidos y otros herbívoros (Ojasti 2000) y ha sido ampliamente utilizado en estudios poblacionales con venado cola blanca en el país (Camargo 2008; Contreras 2008; Coronel et al. 2009; Ezcurra \& Gallina 1981; Galindo-Leal 1993; Galindo-Leal \& Weber 1998; Gallina 1990, 1994; López 2006; Mandujano 1992; Mandujano \& Gallina 1994, 1995; Ortiz-Martínez et al. 2005; Román 1994; Sánchez-Rojas et al. 2009; Valenzuela 1991; Villarreal \& Treviño 1999).

En cada sitio de muestreo se colocó un transecto lineal de $390 \mathrm{~m}$ de largo en el que se establecieron 40 parcelas circulares de $9.3 \mathrm{~m}^{2}$, separadas por intervalos regulares de $10 \mathrm{~m}$. En cada una de ellas se contaron los grupos fecales. Para controlar el tiempo de depósito se realizó un premuestreo en el que se eliminaron todos los grupos fecales presentes en las parcelas. En marzo (temporada de secas) y en junio-julio (temporada de lluvias) de 2008 se realizaron muestreos en cada uno de los transectos. Se definió como grupo fecal a cinco o más pellas encontradas juntas con características físicas similares (tamaño, forma, textura, color y dureza; Mandujano 1992; Ortiz 2000; Román 1994). Se contabilizaron todos los grupos fecales encontrados dentro de la parcela cuando al menos la mitad de las pellas se encontrara dentro de los límites de la parcela (Román 1994).

La densidad poblacional se calculó con el modelo de Eberhardt \& Van Etten (1956), utilizando dos tasas de defecación, debido a que no se conoce esta tasa en los venados de la zona. Se utilizó como el límite inferior la tasa propuesta por Eberhardt \& Van Etten (1956; 12.7 grupos/individuos/día) que es la menor de las tasas conocidas para la especie y la propuesta por Rogers (1987; 33.9 grupos/individuos/día) como límite superior para obtener un intervalo dentro del cual muy probablemente se encuentre la densidad poblacional real.

Caracterización de hábitat. Se caracterizó el hábitat con base en 13 variables de la vegetación y atributos físicos. Las variables de la vegetación fueron: la densidad de árboles, el área basal de los individuos arbóreos, la riqueza de especies, la diversidad calculada a partir del índice de Shannon (Magurran 1988), la altura promedio de los árboles con DAP $\geq 30 \mathrm{~cm}$, la densidad de arbustos y la cobertura de protección horizontal (refugio proporcionado por el hábitat; Griffith \& Youtie 1988; modificado por Ortiz 2000). Las variables físicas fueron: la altitud, la orientación de la ladera, la sinuosidad del terreno (promediando las pendientes determinadas en cada una de las 40 parcelas de cada sitio), la distancia a las fuentes de agua, la temperatura promedio anual y la humedad relativa promedio anual (estas dos últimas variables fueron medidas con ayuda de sensores atmosféricos tipo Hobo ${ }^{\circledR}$ Pro Data Loggers, que registran las condiciones de temperatura y humedad por hora). Para las variables de los estratos arbóreo y arbustivo se tomó información de los trabajos de Aguilar (2007) y Wong (2011). Se tomaron estas variables como medidas de los requerimientos de hábitat 
necesarios para la sobrevivencia de los venados (alimento, agua, cobertura vegetal y espacio vital; Galindo-Leal \& Weber 1998; Villarreal 2006).

Se evaluaron las diferencias significativas de 12 variables medidas entre las cinco asociaciones vegetales presentes en la zona, usando un Análisis de Varianza de una vía o una prueba no paramétrica de Kruskall-Wallis. En los casos en los que se detectaron diferencias significativas se realizaron pruebas post-hoc para determinar la ubicación de tales diferencias (Zar 1999). Los análisis fueron realizados en el programa STATISTICA 7 (StatSoft 2004).

Para identificar las variables del hábitat que determinan la presencia de los venados en la zona se realizó un análisis multivariado con 12 variables de hábitat del venado cola blanca. Se aplicó la técnica de Componentes Principales (APC) para reconocer el aporte de cada variable a la varianza de los componentes principales. Este análisis reduce la dimensionalidad de un conjunto de variables interrelacionadas y al mismo tiempo retiene la mayor parte de la varianza de las mismas (Jolliffe 2002). Debido a que las variables medidas presentan diferencias en la escala, el análisis se realizó con las variables estandarizadas (matriz de correlaciones). Se utilizó un nivel de significancia de $>0.5$ (McGarigal et al. 2000).

\section{RESULTADOS}

Densidad poblacional. En los 13 sitios muestreados (1,011 parcelas) en las dos épocas (secas y lluvias) se encontró un total de 15 grupos fecales, con un promedio de 0.58 grupos/transecto (0.015 grupos/parcela). El tiempo de depósito varió de 61 a 145 días. En promedio, la densidad encontrada en el periodo de marzo a julio de 2008 fue de $1.30 \pm 3.04$ individuos $/ \mathrm{km}^{2}$ (tasa baja) y de $0.49 \pm 1.14$ individuos $/ \mathrm{km}^{2}$ (tasa alta). En el bosque de coníferas, la densidad poblacional estimada fue de $3.17 \pm 4.91$ individuos $/ \mathrm{km}^{2}$ (tasa baja) y de $1.19 \pm 1.84$ individuos $/ \mathrm{km}^{2}$ (tasa alta). Para el bosque de pino-encino cerrado, las densidades poblacionales estimadas fueron de $1.06 \pm 1.57$ individuos $/ \mathrm{km}^{2}$ (tasa baja) y de $0.40 \pm 0.59$ individuos $/ \mathrm{km}^{2}$ (tasa alta; Cuadro 1).

Caracterización de hábitat. Los sitios en el bosque de coníferas se encuentran entre 2,766 y 3,247 m s. n. m. y las especies arbóreas dominantes son Abies hickelii, Pinus patula, $P$. hartwegii y $P$. ayacahuite. La temperatura corresponde a los valores mas bajos de la zona y la humedad relativa es alta, similar a la que se presenta en los sitios ubicados en los bosques de pino-encino cerrados. En promedio, la altura de los árboles en el bosque de coníferas es mayor que en los otros sitios muestreados. Los sitios en los bosques de pino-encino cerrados se distribuyen entre los 2,689 y $2,826 \mathrm{~m} \mathrm{~s}$. $\mathrm{n}$. $\mathrm{m}$. La temperatura media anual fluctúa alrededor de $\operatorname{los} 10^{\circ} \mathrm{C}$. Los árboles dominantes pertenecen a las especies Pinus ayacahuite, P. patula var. longipedunculata, $P$. montezumae, Quercus rugosa y $Q$. laurina. Se encontraron entre 896 a 1,684 árboles/ha con alturas mayores a los 20 metros en promedio. Las distancias a las fuentes de agua están entre los 26 y $72 \mathrm{~m}$. En los sitios del bosque de pino-encino abierto las 
Cuadro 1. Estimaciones de densidad poblacional (ind. $/ \mathrm{km}^{2} \pm$ D. E.) del venado cola blanca en Santa María Yavesía, ordenadas por muestreo y asociación vegetal con las tasas de defecación baja y alta.

\begin{tabular}{lcccccc}
\hline \multirow{2}{*}{$\begin{array}{c}\text { Asociación } \\
\text { vegetal }\end{array}$} & \multicolumn{2}{c}{$\begin{array}{c}\text { Muestreo marzo } \\
\text { (secas) }\end{array}$} & \multicolumn{2}{c}{$\begin{array}{c}\text { Muestreo junio-julio } \\
\text { (lluvias) }\end{array}$} & \multicolumn{2}{c}{ Promedio } \\
\cline { 2 - 7 } & \multicolumn{2}{c}{ Tasa de defecación } & \multicolumn{2}{c}{ Tasa de defecación } & \multicolumn{2}{c}{ Tasa de defecación } \\
\cline { 2 - 7 } & 12.7 & 33.9 & 12.7 & 33.9 & 12.7 & 33.9 \\
\hline $\begin{array}{l}\text { Bosque de } \\
\text { coníferas }\end{array}$ & $2.14 \pm 1.72$ & $0.80 \pm 0.64$ & $4.20 \pm 7.10$ & $1.57 \pm 2.66$ & $3.17 \pm 4.91$ & $1.19 \pm 1.84$ \\
$\begin{array}{l}\text { Bosque de } \\
\text { pino-encino } \\
\text { cerrado }\end{array}$ & $1.02 \pm 2.04$ & $0.38 \pm 0.76$ & $1.10 \pm 1.28$ & $0.41 \pm 0.48$ & $1.06 \pm 1.57$ & $0.40 \pm 0.59$ \\
$\begin{array}{l}\text { Bosque de } \\
\text { pino-encino } \\
\text { abierto }\end{array}$ & 0 & 0 & 0 & & & 0 \\
$\begin{array}{l}\text { Bosque de } \\
\text { encino-pino }\end{array}$ & 0 & 0 & 0 & 0 & 0 & 0 \\
$\begin{array}{l}\text { Encinar } \\
\text { caducifolio }\end{array}$ & 0 & 0 & 0 & 0 & 0 & 0 \\
\begin{tabular}{l} 
Promedio \\
\hline
\end{tabular} & $0.97 \pm 1.62$ & $0.36 \pm 0.61$ & $1.63 \pm 4.05$ & $0.61 \pm 1.52$ & $1.30 \pm 3.04$ & $0.49 \pm 1.14$ \\
\hline
\end{tabular}

especies dominantes son Pinus oaxacana, P. lawsonii, P. patula var. longipedunculata, Quercus crassifolia y $Q$. obtusata. Tienen una altitud de $\operatorname{los} 2,239$ a 2,360 m s. n. $\mathrm{m}$, la humedad relativa es menor (alrededor del 68\%) y tienen una temperatura media anual de $15{ }^{\circ} \mathrm{C}$. En general, la densidad de arbustos es mayor y la altura promedio de los árboles con DAP $\geq 30 \mathrm{~cm}$ es similar a la de los bosques de pino-encino cerrados. El sitio cubierto por bosque de encino-pino está orientado al sureste y las especies dominantes son Quercus laeta, Q. castanea, P. lawsonii y P. oaxacana. Se ubica a $\operatorname{los} 2,405 \mathrm{~m} \mathrm{~s}$. n. m, con temperatura media anual de $15.2^{\circ} \mathrm{C}$ y humedad relativa de $69.2 \%$. En general, los árboles son más bajos que en los bosques descritos antes. Por último, en la parte más baja de la zona se ubica un sitio en el encinar caducifolio, que está compuesto por $Q$. aff. obtusata, $Q$. laeta y P. lawsonii. La altura de los árboles es de $8 \mathrm{~m}$ en promedio (Cuadros 2 y 3 ).

Se encontraron diferencias significativas entre la altura promedio de los árboles con DAP $\geq 30 \mathrm{~cm}$, la altitud, la temperatura promedio anual y la humedad relativa promedio anual. En los cuatro casos, el bosque de coníferas presentó diferencias significativas con las asociaciones vegetales restantes (Cuadro 4).

En el Cuadro 5 se observan los resultados del ACP, que incluye 12 variables del hábitat del venado que presentaron valores significativos $(>0.5)$. En el análisis se retuvieron los primeros tres componentes principales, que capturaron en conjunto el $71 \%$ de la varianza, alcanzando los dos primeros el 58.9\%. Las variables con ma- 
Cuadro 2. Características físicas del hábitat del venado cola blanca en Santa María Yavesía.

\begin{tabular}{|c|c|c|c|c|c|c|}
\hline Sitio & $\begin{array}{c}\text { Altitud } \\
\text { promedio } \\
\text { (m s. n. m.) }\end{array}$ & $\begin{array}{l}\text { Orientación } \\
\text { de la ladera }\end{array}$ & $\begin{array}{l}\text { Sinuosidad } \\
\left({ }^{\circ}\right)\end{array}$ & $\begin{array}{l}\text { Distancia a } \\
\text { fuentes de } \\
\text { agua } \\
\text { (m) }\end{array}$ & $\begin{array}{c}\text { Temperatura } \\
\text { promedio } \\
\text { anual } \\
\left({ }^{\circ} \mathrm{C}\right)\end{array}$ & $\begin{array}{c}\text { Humedad } \\
\text { relativa } \\
\text { promedio anual } \\
(\%)\end{array}$ \\
\hline \multicolumn{7}{|c|}{ Bosque de coníferas } \\
\hline 1 & 3247 & SE & 15 & 67 & 8.1 & 81.66 \\
\hline 2 & 3205 & SW & 29 & 106 & 8.1 & 81.21 \\
\hline 3 & 3067 & NW & 26 & 259 & 9.0 & 81.50 \\
\hline 4 & 2766 & SW & 26 & 223 & 9.5 & 85.61 \\
\hline \multicolumn{7}{|c|}{ Bosque de pino-encino cerrado } \\
\hline 5 & 2826 & SW & 20 & 29 & 10.2 & 81.07 \\
\hline 6 & 2790 & SW & 31 & 72 & 10.0 & 80.00 \\
\hline 7 & 2715 & SW & 16 & 26 & 10.0 & 81.00 \\
\hline 8 & 2689 & NW & 15 & 35 & 10.6 & 79.98 \\
\hline \multicolumn{7}{|c|}{ Bosque de pino-encino abierto } \\
\hline 9 & 2360 & SW & 19 & 225 & 14.9 & 67.00 \\
\hline 10 & 2354 & SW & 23 & 91 & 14.8 & 69.00 \\
\hline 11 & 2239 & NW & 29 & 96 & 15.0 & 68.36 \\
\hline \multicolumn{7}{|c|}{ Bosque de encino-pino } \\
\hline 12 & 2405 & SE & 25 & 140 & 15.2 & 69.20 \\
\hline \multicolumn{7}{|c|}{ Encinar caducifolio } \\
\hline 13 & 2287 & SE & 25 & 88 & 15.5 & 68.00 \\
\hline
\end{tabular}

yor peso en el primer componente se relacionan con el clima (temperatura promedio anual y humedad relativa promedio anual), la altitud, el dosel y el sotobosque; en orden descendente corresponden a la humedad, la temperatura, la altitud, la altura promedio de los árboles con DAP $\geq 30 \mathrm{~cm}$, la cobertura de protección y la densidad de arbustos. La humedad, la altitud y la altura promedio de los árboles con DAP $\geq$ $30 \mathrm{~cm}$ se asociaron de manera positiva, mientras que la temperatura, la densidad de arbustos y la cobertura de protección se asociaron negativamente con este componente. Los sitios 2, 3 y 4 (bosque de coníferas) y, en menor medida, los sitios 5, 6, 7 y 8 (bosque de pino-encino cerrado) presentaron una asociaron positiva con este componente, mientras que los sitios 9, 10, 11 (bosque de pino-encino abierto), 12 (bosque de encino-pino) y 13 (encinar caducifolio), se asociaron de manera negativa. En general, los sitios 2, 3 y 4, que tuvieron las densidades más altas de venados, se caracterizan por tener bajas temperaturas, mayor humedad, mayores altitudes y estratos arbóreo y arbustivo abiertos, pero con una mayor cantidad de árboles grandes (Fig. 2). Las 
Cuadro 3. Variables de la vegetación del hábitat del venado cola blanca en Santa María Yavesía (datos modificados de Aguilar, 2007; Wong, 2011).

\begin{tabular}{|c|c|c|c|c|c|c|c|}
\hline Sitio & $\begin{array}{l}\text { Densidad } \\
\text { de árboles } \\
\text { (ind./ha) }\end{array}$ & $\begin{array}{l}\text { Densidad } \\
\text { de } \\
\text { arbustos } \\
\text { (ind./ha) }\end{array}$ & $\begin{array}{l}\text { Área basal } \\
\left(\mathrm{m}^{2}\right)\end{array}$ & $\begin{array}{c}\text { Altura } \\
\text { promedio de } \\
\text { árboles } \geq 30 \\
\text { cm DAP } \\
\text { (m) }\end{array}$ & $\begin{array}{l}\text { Riqueza } \\
\text { de } \\
\text { especies }\end{array}$ & $\begin{array}{c}\text { Índice de } \\
\text { diversidad } \\
\text { de Shannon } \\
\left(H^{\prime}\right)\end{array}$ & $\begin{array}{l}\text { Cobertura de } \\
\text { protección } \\
\text { horizontal } \\
\text { promedio } \\
(\%)\end{array}$ \\
\hline \multicolumn{8}{|c|}{ Bosque de coníferas } \\
\hline 1 & 1137 & 624 & 24.0 & 20.9 & 6 & 0.34 & 13.5 \\
\hline 2 & 867 & 55 & 44.0 & 26.6 & 10 & 0.86 & 26.5 \\
\hline 3 & 987 & 187 & 66.3 & 32.9 & 23 & 2.44 & 41.5 \\
\hline 4 & 663 & 72 & 52.3 & 28.8 & 15 & 2.05 & 27.0 \\
\hline \multicolumn{8}{|c|}{ Bosque de pino-encino cerrado } \\
\hline 5 & 982 & 194 & 35.8 & 20.4 & 14 & 1.96 & 63.0 \\
\hline 6 & 1002 & 168 & 36.2 & 22.6 & 9 & 1.68 & 52.5 \\
\hline 7 & 1684 & 194 & 35.2 & 22.3 & 12 & 1.72 & 46.5 \\
\hline 8 & 896 & 244 & 28.5 & 21.4 & 18 & 2.29 & 58.0 \\
\hline \multicolumn{8}{|c|}{ Bosque de pino-encino abierto } \\
\hline 9 & 2460 & 414 & 16.9 & 19.3 & 9 & 1.65 & 85.0 \\
\hline 10 & 710 & 105 & 54.3 & 21.3 & 9 & 1.47 & 32.5 \\
\hline 11 & 1497 & 334 & 45.0 & 27.9 & 15 & 2.01 & 31.5 \\
\hline \multicolumn{8}{|c|}{ Bosque de encino-pino } \\
\hline 12 & 910 & 183 & 16.5 & 16.4 & 8 & 1.95 & 68.5 \\
\hline \multicolumn{8}{|c|}{ Encinar caducifolio } \\
\hline 13 & 1698 & 520 & 62.7 & 7.7 & 14 & 1.68 & 61.0 \\
\hline
\end{tabular}

variables con mayor peso en el segundo componente se encuentran relacionadas con la diversidad, el área basal de los árboles, el relieve y la disponibilidad de agua; en orden descendente se encontraron la diversidad, la riqueza de especies, el área basal de los árboles, la sinuosidad y la distancia a fuentes de agua. Todas las variables se asociaron de manera negativa a este componente. El sitio 1, en el que no se registró presencia de venados, se asoció de manera positiva con este componente. Este sitio presentó baja riqueza de especies arbóreas y baja diversidad. En el tercer componente, la variable de mayor peso fue la sinuosidad, asociada a este componente de manera positiva.

De acuerdo al ACP, los sitios de muestreo se dividieron en cuatro grupos. En el primero se ubican tres sitios en el bosque de coníferas que presentaron la mayor densidad de venados; en el segundo, los sitios con bosque de pino-encino cerrado, que 
Cuadro 4. Resultados de las pruebas estadísticas realizadas a las variables del hábitat del venado cola blanca en Santa María Yavesía (*Diferencia significativa: $\mathrm{p}<0.05$ ).

\begin{tabular}{lccc}
\hline \multicolumn{1}{c}{ Variables del hábitat } & $\mathrm{F}$ & $\mathrm{H}$ & $\mathrm{p}$ \\
\hline Densidad de árboles & - & 3.34 & 0.50 \\
Densidad de arbustos & - & 2.71 & 0.61 \\
Área basal & 1.61 & - & 0.26 \\
Altura promedio de árboles $\geq 30$ cm DAP & 5.91 & - & $0.02^{*}$ \\
Riqueza de especies & 0.31 & - & 0.86 \\
Índice de diversidad de Shannon & - & 1.08 & 0.90 \\
Cobertura de protección horizontal promedio & 2.08 & - & 0.18 \\
Altitud & 34.08 & - & $<0.01 *$ \\
Sinuosidad (grados) & 0.24 & - & 0.91 \\
Distancia promedio a fuentes de agua & - & 7.55 & 0.11 \\
Temperatura promedio anual & 120.53 & - & $<0.01 *$ \\
Humedad relativa promedio anual & - & 11.06 & $0.03^{*}$ \\
\hline
\end{tabular}

también tuvieron presencia de venados, pero en menor magnitud; un tercero, con los sitios en el bosque de pino-encino abierto, bosque de encino-pino y encinar caducifolio, en los que no se obtuvieron registros y un cuarto, con un sitio en el bosque de coníferas en el que tampoco se observó la presencia de venados (Fig. 2).

\section{DISCUSIÓN}

La densidad poblacional estimada en el periodo analizado para el venado cola blanca de Santa María Yavesía fue relativamente baja si se le compara con otros estudios realizados en el país con el método de conteo de grupos fecales. Sin embargo, la densidad encontrada en el bosque de coníferas y en el bosque de pino-encino fue mayor a la reportada en un trabajo realizado durante 1998 y 1999 en "Pueblos Mancomunados", zona que incluye a Santa María Yavesía (Ortiz-Martínez et al. 2005; Cuadro 6).

Es importante notar que en ambos trabajos la mayor densidad de venados fue encontrada en sitios con bosques de coníferas, que se ubican a mayores altitudes. Tanto en el trabajo de Ortiz-Martínez et al. (2005), como en este trabajo, se reportaron disturbios antropogénicos derivados del manejo forestal. En particular, durante la realización de este trabajo se llevaron a cabo actividades de saneamiento forestal en la zona, que derivaron en la presencia constante de trabajadores, introducción de maquinaria y camiones de carga. Asimismo, Ortiz-Martínez et al. (2005) reportan perturbaciones ocasionadas por el tránsito de vehículos y trabajadores, así como ruido producido por el aprovechamiento forestal. Estos factores podrían afectar la pre- 
Piña \& Trejo: Densidad y hábitat del venado cola blanca en Oaxaca

Cuadro 5. Resultados del Análisis de Componentes Principales (ACP) realizado a las variables del hábitat del venado cola blanca en Santa María Yavesía.

\begin{tabular}{lccc}
\hline \multicolumn{1}{c}{ Componente } & I & II & III \\
\hline Varianza explicada & 35.5 & 23.4 & 12.1 \\
Varianza acumulada & 35.5 & 58.9 & 71.0 \\
VARIABLE & & & \\
Estrato arbóreo & & & \\
Altura promedio de árboles con DAP $\geq 30 \mathrm{~cm}$ & 0.7649 & -0.2899 & -0.0501 \\
Densidad de árboles & -0.6900 & 0.0648 & -0.2726 \\
Área basal & 0.3700 & -0.5680 & 0.3078 \\
Estrato arbustivo & & & \\
Densidad de arbustos & -0.5056 & 0.4500 & -0.1272 \\
Cobertura de protección $(\%$ promedio) & -0.6781 & -0.2503 & -0.4700 \\
Diversidad & & & \\
Riqueza de especies & 0.3513 & -0.6839 & -0.4702 \\
Diversidad & -0.0415 & -0.8375 & -0.4540 \\
Atributos físicos & & & \\
Distancia a fuentes de agua (m) & & -0.5302 & -0.0113 \\
Altitud (m s. n. m.) & 0.1045 & 0.4560 & -0.1516 \\
Sinuosidad $\left(^{\circ}\right)$ & 0.8062 & -0.5337 & 0.6555 \\
Temperatura $\left(^{\circ}\right)$ & 0.1916 & -0.3710 & 0.2573 \\
Humedad (mm) & -0.8688 & 0.2374 & -0.3189 \\
\hline
\end{tabular}

sencia, así como las preferencias de hábitat, de los venados en la zona, lo que puede ocasionar cambios en la distribución de los venados, obligándolos a refugiarse en los sitios más altos e inaccesibles, lejos de los cazadores, trabajadores, maquinaria y vehículos (Dorrance et al. 1975; Kilgo et al. 1998; Kufeld et al. 1988; Rost \& Bailey 1979; Swenson 1982), aún si estos sitios no presentan condiciones óptimas que favorecen la presencia de los venados cola blanca (p. ej., sotobosque más desarrollado, mayor sinuosidad). En otras zonas del país con actividades de manejo forestal o con ganadería se ha encontrado que el venado cola blanca puede desplazarse a hábitats con mayor altitud (Hernández et al. 2011; Medina et al. 2008), como una estrategia para evitar estos factores de disturbio.

No obstante, en los bosques de coníferas de este trabajo se encontraron densidades superiores a las reportadas por Ortiz-Martínez et al. (2005) en la misma asociación vegetal (bosque de oyamel-pino), lo que podría deberse a que durante la realización de dicho estudio se reportaron disminuciones en la productividad de especies arbustivas de las que depende la alimentación del venado cola blanca en la zona, esto debido 


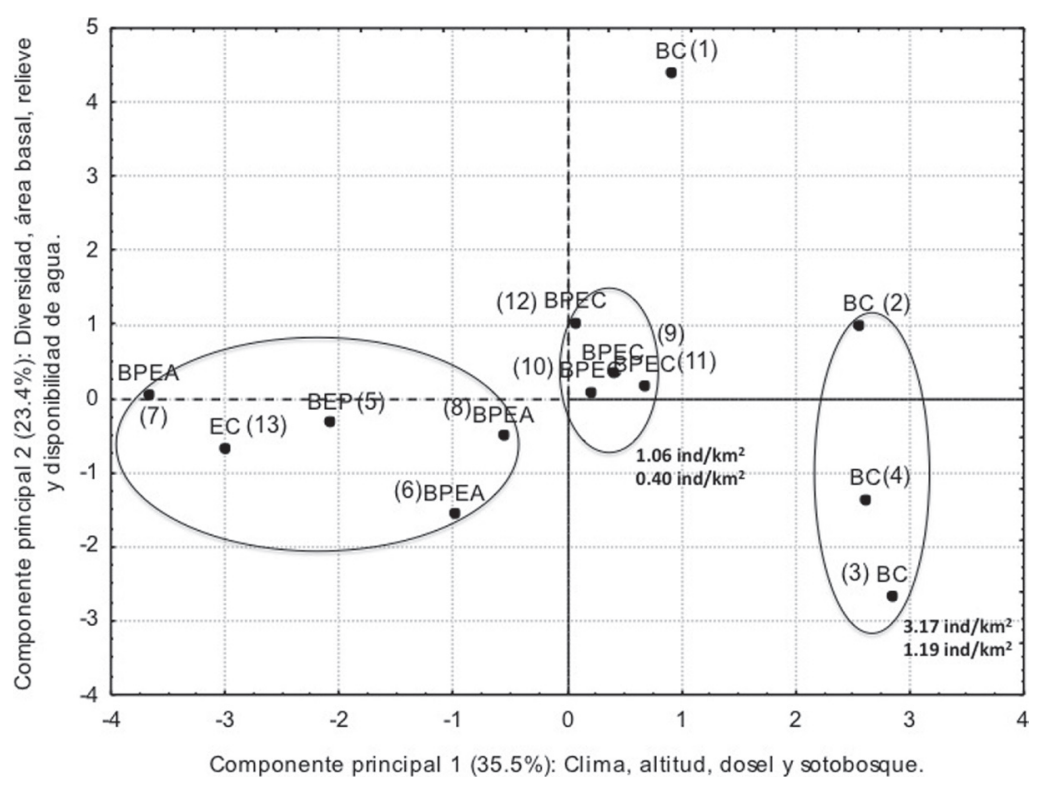

Figura 2. Posición de los sitios pertenecientes al bosque de coníferas (BC: 1, 2, 3 y 4), bosque de encino-pino (BEP: 5), bosque de pino-encino abierto (BPEA: 6, 7 y 8), bosque de pino-encino cerrado (BEPC: $9,10,11$ y 12) y encinar caducifolio (EC: 13) con relación a los componentes principales 1 y 2. Los círculos representan los grupos en los que se asociaron los transectos (BC, BPEC y BPEA/BEP/ EC). El sitio 1 (bosque de coníferas) no mostró relación con los demás sitios. Se presentan los valores de densidad (ind. $/ \mathrm{km}^{2}$ ) encontrados por asociación vegetal. Se incluyen los valores con las dos tasas de defecación utilizadas (12.7 y 33.9 grupos/ind./día).

a una marcada temporada de sequía en 1998, lo que pudo haber tenido efectos negativos sobre el tamaño poblacional de los venados de la zona. Esto es consistente con lo reportado aquí, ya que se encontró que aunque los bosques de coníferas presentan un sotobosque abierto (con respecto de los sitios muestreados en las demás asociaciones vegetales), presentan área basal y densidad de árboles y arbustos mayor que en lo reportado en los sitios con bosque de coníferas de Ortiz-Martínez et al. (2005), hecho que sugiere que los bosques de coníferas se están recuperando de la temporada de sequía de 1998 y tienen el potencial de actuar como refugios para el venado cola blanca, al menos mientras las actividades de caza y manejo forestal continúen desarrollándose en el resto de la zona.

La tasa de defecación alta (33.9 grupos/individuos/día) empleada en este trabajo fue mayor que las tasas utilizadas en todos los trabajos referidos, lo cual podría dar como resultado que la densidad poblacional de los venados calculada para la zona se esté subestimando. En el caso contrario, al utilizar la tasa de defecación baja (12.7 grupos/individuos/día) es probable que el resultado obtenido sobreestime la densi- 
dad de la población. Es por ello que para estimar con mayor precisión la densidad poblacional con el conteo de grupos fecales es necesario que se establezca la tasa de defecación para los venados de la zona de estudio (Mandujano \& Gallina 1994; Pérez-Mejía et al. 2004). Esto de suma importancia, ya que pequeños errores en las estimaciones de las cosechas sustentables pueden derivar en la sobreexplotación y/o la extirpación del venado cola blanca (Mandujano 2007) y los datos obtenidos de estudios poblacionales con este método podrían ser utilizados como base para el manejo sustentable de la especie en las comunidades aledañas.

El método de conteo de grupos fecales tiene la ventaja de que no es necesario observar a los venados de manera directa, por lo que se causan pocos disturbios a la población y se pueden obtener tamaños de muestras mayores que con otros métodos (Ezcurra \& Gallina 1981; Mandujano \& Gallina 1994). Para obtener información confiable es necesario contar con información de la biología de la especie en la zona de estudio, como el tipo de dieta (relacionado con el tipo de hábitat), la estación del año, el sexo, tomar en cuenta que los venados tienden a defecar en sitios específicos (usualmente cerca de sus lugares de descanso) y las condiciones en las que se determinaron estas tasas (animales en cautiverio o en vida libre, horas de observación, número de animales observados) para establecer la tasa de defecación (Mandujano \& Gallina 1994, 1995; Rogers 1987).

Recomendaciones de manejo. Los esfuerzos para el manejo y la conservación de los venados de la zona deben estar enfocados a fomentar su uso racional. Es crucial que se implementen estrategias que conduzcan a la conservación y manejo integral de los bosques y la fauna de la comunidad (Ambriz 2003). Se ha reconocido que la cacería no regulada puede ser la principal causa de la escasez de venados en los bosques templados (Galindo-Leal \& Weber 1998), así como de la modificación de sus preferencias de hábitat (Reyna-Hurtado \& Tanner 2005). Aún en un hábitat propicio para sostener a los venados, pueden encontrarse densidades muy bajas o, incluso, ausencia de la especie, debido a costumbres de aprovechamiento negativas que en muchas ocasiones se encuentran fuertemente arraigadas en las poblaciones locales.

Este trabajo contribuye con el conocimiento del venado cola blanca en el área; sin embargo, es necesario ampliar los estudios, continuar con las estimaciones poblacionales e implementar un programa de monitoreo a largo plazo en un mayor número de sitios para poder obtener valores de densidad más precisos, así como para definir las tendencias poblacionales de la especie en la zona (Ortiz-Martínez et al. 2005). De igual forma, sería importante analizar la estructura y dinámica poblacional de la especie, información básica si se pretende en un futuro realizar acciones de manejo (Gallina 1990). Además, se debe profundizar en la identificación de los hábitos alimentarios de los venados en la zona, así como determinar con precisión las características del hábitat que favorecen o limitan su presencia (Galindo-Leal 1993; Weber et al. 2006). 
Cuadro 6. Densidades poblacionales del venado cola blanca estimadas con el método de conteo de grupos fecales en bosques templados de México.

\begin{tabular}{|c|c|c|c|c|}
\hline Estudio & $\begin{array}{l}\text { Densidad } \\
\text { (ind. } / \mathrm{km}^{2} \text { ) }\end{array}$ & $\begin{array}{c}\text { Tasa de } \\
\text { defecación } \\
\text { (grupos/ind./día) }\end{array}$ & $\begin{array}{c}\text { Tipo de } \\
\text { vegetación }\end{array}$ & Localidad \\
\hline \multirow[t]{4}{*}{ Este trabajo } & $3.17 \pm 4.91$ & 12.7 & $\begin{array}{l}\text { Bosque de } \\
\text { coníferas }\end{array}$ & \multirow{4}{*}{$\begin{array}{l}\text { Santa María } \\
\text { Yavesía, Sierra } \\
\text { Norte, Oaxaca }\end{array}$} \\
\hline & $1.06 \pm 1.57$ & 12.7 & $\begin{array}{l}\text { Bosque de } \\
\text { pino-encino }\end{array}$ & \\
\hline & $1.19 \pm 1.84$ & 33.9 & $\begin{array}{l}\text { Bosque de } \\
\text { coníferas }\end{array}$ & \\
\hline & $0.40 \pm 0.59$ & 33.9 & $\begin{array}{l}\text { Bosque de } \\
\text { pino-encino }\end{array}$ & \\
\hline $\begin{array}{l}\text { Ortiz-Martínez et } \\
\text { al. } 2005\end{array}$ & $1.13 \pm 1.15$ & 12.7 & $\begin{array}{l}\text { Bosques } \\
\text { templados }\end{array}$ & $\begin{array}{l}\text { Pueblos } \\
\text { Mancomunados, } \\
\text { Sierra Norte, } \\
\text { Oaxaca }\end{array}$ \\
\hline $\begin{array}{l}\text { Sánchez-Rojas et } \\
\text { al. } 2009\end{array}$ & $2.10 \pm 1.60$ & 12.7 & $\begin{array}{l}\text { Bosque de pino, } \\
\text { bosque de encino } \\
\text { y pastizal }\end{array}$ & $\begin{array}{l}\text { Huasca de } \\
\text { Ocampo, Hidalgo }\end{array}$ \\
\hline Valenzuela 1991 & $4.83 \pm 0.98$ & 12.7 & $\begin{array}{l}\text { Bosque de } \\
\text { pino-encino }\end{array}$ & $\begin{array}{l}\text { La Primavera, } \\
\text { Jalisco }\end{array}$ \\
\hline \multirow[t]{2}{*}{ Román 1994} & 1.09 & & $\begin{array}{l}\text { Bosque de } \\
\text { encino-pino }\end{array}$ & \multirow{2}{*}{$\begin{array}{l}\text { Bosques } \\
\text { montanos de } \\
\text { Jalisco y Colima }\end{array}$} \\
\hline & 7.94 & & $\begin{array}{l}\text { Bosque de } \\
\text { pino-encino }\end{array}$ & \\
\hline Gallina 1990 & $0.002 \pm 0.0002$ & 12.7 & $\begin{array}{l}\text { Bosque de } \\
\text { pino-encino }\end{array}$ & $\begin{array}{l}\text { Reserva de la } \\
\text { Biósfera La } \\
\text { Michilía, Durango }\end{array}$ \\
\hline \multirow[t]{2}{*}{$\begin{array}{l}\text { Galindo-Leal y } \\
\text { Weber } 1998\end{array}$} & 5.90 & 12.7 & \multirow[t]{2}{*}{$\begin{array}{l}\text { Bosque de } \\
\text { pino-encino }\end{array}$} & $\begin{array}{l}\text { Reserva de la } \\
\text { Biósfera La }\end{array}$ \\
\hline & 3.40 & 22.0 & & Michilía, Durango \\
\hline
\end{tabular}

Asimismo, para lograr un manejo adecuado de las poblaciones de la especie en la zona es necesario determinar cuotas de caza y épocas de veda, para lo cual se deben analizar las tasas de aprovechamiento, tomando como base la calidad y capacidad de carga del hábitat para el año en cuestión, las condiciones climatológicas presentes y anteriores, la densidad poblacional media, la relación machos:hembras y hembras adultas:cervatos, la carga animal y el manejo de otros animales que compartan el hábitat (p. ej. ganado), así como el grado de afectación por la depredación y los registros de caza que incluyan, al menos, la edad, el sexo y las medidas de las canastas de astas 
de los animales cazados en temporadas anteriores. Asimismo, es recomendable la extracción conjunta de machos y hembras, ya que con esto se logra controlar la densidad y se mantiene la estructura de la población (Gallina 1990; Villarreal 2006).

La administración de cualquier estrategia de manejo debe estar regulada por los pobladores locales. Esto debido a que la consideración de los miembros de una comunidad como actores sociales participantes, y no sólo como proveedores del espacio que mantiene la vida silvestre, repercute en las capacidades sociales de las comunidades, permitiéndoles controlar el uso de sus recursos naturales y, por lo tanto, corresponsabilizándolas de su mantenimiento, esto para su propio beneficio y para la conservación de la vida silvestre (García 2005). Asimismo, los pobladores de las comunidades indígenas, dueños directos de los bosques, deben fungir como los principales actores en la conservación de las especies animales y que sin su convencimiento, cualquier estrategia planteada desde otros grupos de la sociedad seguramente no logrará los objetivos de conservación integral (González et al. 2004).

Es recomendable constituir un comité de vigilancia designado por la misma comunidad que apoye la protección de la especie para que pueda recuperarse (Briones 1997; Ortiz-Martínez et al. 2005); implementar talleres participativos en los que se intercambien conocimientos sobre la especie, su uso tradicional y para que se promueva una actitud a favor del aprovechamiento sustentable en las costumbres de caza (Ortiz-Martínez et al. 2005); capacitar a los pobladores para que participen directamente en el manejo de la especie y vedas autoimpuestas para aumentar la densidad poblacional de los venados de la zona, hasta que se demuestre que la población se ha incrementado. Esto permitirá construir un programa de aprovechamiento de autoconsumo y con fines cinegéticos (Briones 1997; López 2006; López-Téllez et al. 2007).

Existen varios esquemas de manejo sustentable que podrían ser implementados en la zona, entre los que se encuentran las Unidades de Manejo y Aprovechamiento de la Vida Silvestre (UMA), las Áreas Comunitarias Protegidas y el Programa de Tierras para la Conservación (Piña 2010). Aunque las UMA constituyen el único esquema de manejo y aprovechamiento sustentable reconocido por la Legislación Mexicana y se encuentran organizadas en un sistema (SUMA), presentan una serie de ventajas y desventajas que deben tomarse en cuenta antes de que puedan ser establecerse en cualquier zona del país (Diario Oficial de la Federación 2006, 2007; García 2005; García-Marmolejo et al. 2008; González et al. 2003; Mandujano \& González-Zamora 2009; SEMARNAT 2000, 2009a, b; Weber et al. 2006). Por otro lado, las Áreas Comunitarias Protegidas y las Tierras para la Conservación constituyen opciones de manejo alternativas a las UMA, pero no están reconocidas formalmente por la Legislación Mexicana (González et al. 2004; Gutiérrez et al. 2002; Pronatura 2009; WWF 2003).

Se ha propuesto a nivel nacional la creación de un "Grand Slam" (torneo de caza deportiva) de venado cola blanca mexicano para crear la demanda de trofeos cine- 
géticos para todas las subespecies mexicanas, de tal manera que si se incrementa el número de UMA en las diferentes regiones de México, se estimularía la competencia entre los cazadores para lograr los mejores trofeos de cada subespecie, evitando su extinción, a la par que se podrían generan ingresos adicionales en las comunidades rurales del país (Villarreal 2006; Villarreal-Espino 2002).

Se considera que además de la caza, en la comunidad existe un gran potencial ecoturístico para la apreciación del venado cola blanca (observación y fotografía) y que el aumento de sus poblaciones podría favorecer dicha actividad, que se traduciría en la obtención de ingresos económicos adicionales, a la par que se fomenta la educación ambiental entre los turistas.

En este trabajo se analizó la densidad poblacional y se caracterizó el hábitat de una subespecie rara y poco estudiada de venado cola blanca en la comunidad de Santa María Yavesía, Oaxaca, en la cual existe potencial para su aprovechamiento. Las recomendaciones de manejo aquí mencionadas deberán tomarse en cuenta para la protección, así como para el aprovechamiento sustentable de la especie.

Agradecimientos. El presente trabajo se llevó a cabo con apoyo económico que recibió E. Piña como becaria del Consejo Nacional de Ciencia y Tecnología (CONACyT), de agosto de 2006 a julio de 2008 (registro 202650). El trabajo formó parte del proyecto IN227308-2 “Contribución a la Construcción de Alternativas Dirigidas a la Conservación de los Bosques de la Cuenca Alta del Río Papaloapan, Oaxaca, México" otorgado por el Programa de Apoyo a Proyectos de Investigación e Innovación Tecnológica (PAPIIT). A J. Wong y A. Aguilar por proporcionar información esencial para la realización de este trabajo. A J. Wong, E. Orta, P. Montes, F. Rojas, A. Pérez, R. Cruz, Y. Medina, G. Cerón, P. Arias, A. Albuerne y F. Azpeitia por su colaboración en el trabajo de campo.

\section{LITERATURA CITADA}

Aguilar, A. 2007. Estructura del bosque templado en la comunidad de Santa María Yavesía, en la Sierra de Juárez, Oax., México. Tesis de Licenciatura. Facultad de Ciencias, UNAM.

Ambriz, G. 2003. Análisis faunístico de los mamíferos de los bosques de Santa María Yavesía, Ixtlán, Oaxaca. Tesis de Licenciatura. Facultad de Ciencias, UNAM.

Aranda, M. 2000. Huellas y otros rastros de los mamíferos grandes y medianos de México. CONABIOInstituto de Ecología, A. C. Xalapa, Veracruz.

Argüeyes, L. M. 2008. UMAs extensivas de venado colablanca como instrumento económico de restauración ecológica en el noroeste de Morelos. Tesis de Maestría. Posgrado en Ciencias Biológicas. UNAM.

Benítez, E. E. 2006. Estructura, composición y diversidad beta en un gradiente altitudinal de los bosques de la comunidad de Santa María Yavesía, Oaxaca. Tesis de Licenciatura. Facultad de Ciencias, UNAM.

Briones. M. 1997. Manejo y conservación del venado cola blanca en comunidades indígenas de la Sierra Norte de Oaxaca. Memorias del $2^{\circ}$ Foro Estatal de Investigación Científica y Tecnológica: "El impacto de la investigación y el desarrollo tecnológico en la problemática estatal. 4 y 5 de diciembre de 1997, pp. 400-403. 
Briones-Salas, M. \& García, C. 2005. Estimación de la densidad del venado cola blanca (Odocoileus virginianus oaxacensis) en la Sierra Norte de Oaxaca. Revista Mexicana de Mastozoología, 9: 141145.

Camargo, A. A. 2008. Evaluación del conteo de grupos fecales y del análisis morfométrico de pellets como métodos de obtención de parámetros demográficos del venado cola blanca (Odocoileus virginianus mexicanus) en Puebla, México. Tesis de Maestría. Instituto Nacional de Ecología. Xalapa, Veracruz.

Contreras, F. M. 2008. Ecología poblacional del venado cola blanca (Odocoileus virginianus thomasi) en la R/A San Joaquín Municipio de Balancán, Tabasco, México. Tesis de Licenciatura. Universidad Juárez Autónoma de Tabasco.

Coronel, H., López, C. A. \& Moreno, C. N. 2009. ¿Pueden las variables de paisaje predecir la abundancia de venado cola blanca? El caso del noroeste de México. Tropical Conservation Science, 2: 229-236.

Dasmann, W. 1971. If deer are to survive. Stackpole Books, Harrisburg, PA, USA. 127 pp.

De Fries, R., Pagiola, S., Adamowicz, W. L., Akçakaya, H. R., Arcenas, A., Babu, S., Balk, D., Confalonieri, U., Cramer, W., Falconí, F., Fritz, S., Green, R., Gutiérrez-Espeleta, E., Hamilton, K., Kane, R., Latham, J., Matthews, E., Ricketts, T. \& Xiang Yue, T. 2005. Analytical approaches for assessing ecosystem condition and human wellbeing. In: R. Hassan, R. Scholes \& N. Ash (Eds.). Millennium Ecosystem Assessment. Current state and trends assessment. Vol. 1. Island Press, Washinghton, D. C. pp: 37-71.

Diario Oficial de la Federación. 2006. Reglamento de la Ley General de Vida Silvestre. SEMARNAT. México, D. F.

Diario Oficial de la Federación. 2007. Ley General de Vida Silvestre. Cámara de Diputados del H. Congreso de la Unión. Centro de Documentación, Información y Análisis. México, D. F.

Dorrance, M. J., Savage, P. J. \& Huff, D. E. 1975. Effects of snowmobiles on white-tailed deer. Journal of Wildlife Management, 39: 563-569.

Eberhardt, L. \& Van Etten, R. C. 1956. Evaluation of the pellet group counts as a deer census method. Journal of Wildlife Management, 20: 70-74.

Ezcurra, E. \& Gallina, S. 1981. Biology and population dynamics of white-tailed deer in northwestern Mexico. In: Folliott, P. F. \& S. Gallina (Eds.). Deer biology, habitat requirements and management in western North America. Instituto de Ecología, A. C. México, D. F., pp. 77-108.

Foster, R. J., Harmsen, B. J., Valdes, B., Pomilla, C. \& Doncaster, C. P. 2009. Food habits of sympatric jaguars and pumas across a gradient of human disturbance. Journal of Zoology, 280: 309-318.

Galindo-Leal, C. 1993. Densidades poblacionales de los venados cola blanca, cola negra y bura en Norteamérica. In: Medellín, R. \& G. Ceballos (Eds.). 1993. Avances en el estudio de los mamíferos de México. Asociación Mexicana de Mastozoología, A. C. Publicaciones Especiales. Vol. I. México, D. F., pp. 371-391.

Galindo-Leal, C. \& Weber, M. 1998. El venado de la Sierra Madre Occidental: Ecología, conservación y manejo. Edicusa-CONABIO. México.

Gallina, S. 1990. El venado cola blanca y su hábitat en La Michilía, Dgo. Tesis de Doctorado. Facultad de Ciencias, UNAM. México, D. F.

Gallina, S. 1994. Dinámica poblacional y manejo de la población del venado cola blanca en la Reserva de la Biosfera la Michilia, Durango, México. In: Vaughan, C. \& M. Rodríguez (Eds.). Ecología y manejo del venado cola blanca en México y Costa Rica. Editorial de la Universidad Nacional de Costa Rica., pp. 207-234.

García, G. 2005. Caracterización y sustentabilidad de las Unidades para la Conservación, Manejo y Aprovechamiento Sustentable de la Vida Silvestre en Campeche. Tesis de Maestría. El Colegio de la Frontera Sur. Campeche, México. 
García-Marmolejo, G., Escalona-Segura, G. \& Van Der Wal, H. 2008. Multicriteria evaluation of Wildlife Management Units in Campeche, Mexico. Journal of Wildlife Management, 72: 11941204.

González, G. \& Briones-Salas, M. 2000. El venado cola blanca (Odocoileus virginianus) en comunidades indígenas de Oaxaca. Investigación Hoy, 94: 20-27.

González, G., Briones-Salas, M. \& Alfaro, A. M. 2004. Integración al conocimiento faunístico del estado. In: García-Mendoza, A., M. J. Ordóñez \& M. Briones-Salas (Eds.). Biodiversidad de Oaxaca. Instituto de Biología, UNAM-Fondo Oaxaqueño para la Conservación de la Naturaleza-WWF. México., pp. 449-466.

González, R. M., Montes, R. \& Santos, J. 2003. Caracterización de las Unidades para la Conservación, Manejo y Aprovechamiento Sustentable de la Fauna Silvestre en Yucatán, México. Tropical and Subtropical Agroecosystems, 2: 13-21.

Griffith, B. \& Youtie, B. A. 1988. Two devices for estimating foliage density and deer hiding cover. Wildlife Society Bulletin, 16: 206-210.

Gutiérrez, M., Bacmeister, A., Ortiz, G. \& Montesinos, J. A. 2002. Herramientas legales para la conservación de tierras privadas y sociales en México. Pronatura, A. C. México, D. F.

Hall, E. R. 1981. The mammals of North America. 2a ed. John Wiley and Sons. Vol. II.

Halls, L. K. 1984. White-tailed deer. Ecology and management. Stackpole Books-Wildlife Management Institute. Pennsylvania.

Hernández, D. A., Cortés, E., Zaragoza, J. L., Martínez, P. A., González, G. T., Rodríguez, B. \& Hernández, D. A. 2011. Hábitat del venado cola blanca en la Sierra de Huautla, Morelos, México. Acta Zoológica Mexicana (n. s.), 27: 47-66.

Jiménez, J. L. 2004. Estructura del bosque de Abies hickelii Flous et Gaussen en la comunidad de Santa María Yavesía, Distrito de Ixtlán, Oaxaca, México. Tesis de Licenciatura. Facultad de Ciencias, UNAM. México, D. F.

Jolliffe, I. T. 2002. Principal Component Analysis. $2^{\text {a }}$ ed. Springer-Verlag. Nueva York.

Kilgo, J. C., Labisky, R. F. \& Fritzen, D. E. 1998. Influences of hunting on the behavior of white-tailed deer: implications for conservation of the Florida panther. Conservation Biology, 12: 1359-1364.

Kufeld, R. C., Bowden, D. C. \& Schrupp, D. L. 1988. Influence of hunting on movements of female mule deer. Journal of Range Management, 41: 70-72.

Leopold, A. S. 1977. Fauna silvestre de México. Aves y mamíferos de caza. Instituto Mexicano de Recursos Naturales Renovables. México, D.F.

López, M. C. 2006. Análisis de la densidad poblacional y caracterización del venado cola blanca (Odocoileus virginianus mexicanus) en cuatro localidades del Municipio de Jolalpan, Puebla. Tesis de Maestría. Facultad de Ciencias, UNAM.

López-Téllez, M. C., Mandujano, S. \& Yánes, G. 2007. Evaluación poblacional del venado cola blanca en un bosque tropical seco de la Mixteca Poblana. Acta Zoológica Mexicana (n. s.), 23: 1-16.

Magurran, A. E. 1988. Ecological diversity and its measurement. Princeton University Press. Princeton.

Mandujano, S. 1992. Estimaciones de la densidad del venado cola blanca (Odocoileus virginianus) en un bosque tropical caducifolio de Jalisco. Tesis de Maestría. Facultad de Ciencias, UNAM. México.

Mandujano, S. 2004. Análisis bibliográfico de los estudios de venados en México. Acta Zoológica Mexicana (n. s.), 20: 211-251.

Mandujano, S. 2007. Carrying capacity and potential production of ungulates for human use in a Mexican tropical dry forest. Biotropica, 39: 519-524.

Mandujano, S. \& Gallina, S. 1994. Comparación de métodos para estimar la densidad poblacional del venado cola blanca en un bosque tropical caducifolio de México, pp. 263-280. In: Vaughan, C. \& 
M. Rodriguez (Eds.). Ecología y manejo del venado cola blanca en México y Costa Rica. Editorial de la Universidad Nacional, Heredia, Costa Rica.

Mandujano, S. \& Gallina, S. 1995. Comparison of deer censusing methods in tropical dry forest. Wildlife Society Bulletin, 23: 180-186.

Mandujano, S. \& González-Zamora, A. 2009. Evaluation of Natural Conservation Areas and Wildlife Management Units to support minimum viable populations of white-tailed deer in Mexico. Tropical Conservation Science, 2: 237-250.

Mandujano, S. \& Rico-Gray, V. 1991. Hunting, use, and knowledge of the biology of the white-tailed deer (Odocoileus virginianus Hays) by the Maya of Central Yucatán, Mexico. Journal of Ethnobiology, 11: 175-183.

Martínez, A., Hewitt, D. G. \& Cotera, M. 1997. Managing overabundant White-tailed deer in Northern Mexico. Wildlife Society Bulletin, 25: 430-432.

McCullough, D. R. 1987. The theory and management of Odocoileus populations, pp. 535-549. In: Wemmer, C. M. (Ed.). Biology and management of the Cervidae: a conference held at the Conservation and Research Center, National Zoological Park, Smithsonian Institution. Front Royal, Virginia, Agosto 1-5, 1982. Series: Research Symposia of the National Zoological Park. Smithsonian Institution. Washington.

McGarigal, K., Cushman, S. \& Stafford, S. 2000. Multivariate statistics for wildlife and ecology research. Springer-Verlag. Nueva York.

Medina, S. M., García, E., Márquez, M., Vaquera, H., Romero, A. \& Martínez, M. 2008. Factores que influyen en el uso del hábitat por el venado cola blanca (Odocoileus virginianus couesi) en la Sierra del Laurel, Aguascalientes, México. Acta Zoológica Mexicana (n. s.), 24: 191-212.

Núñez, R., Miller, B. \& Lindzey, F. 2002. Ecología del jaguar en la reserva de la biósfera ChamelaCuixmala, Jalisco, México, pp. 107-126. In: Medellín, R. A., C. Equihua, C. L. B. Chetkiewicz, P. G. Crawshaw, A. Rabinowitz, K. H. Redford, J. G. Robinson, E. W. Sanderson \& A. B. Taber (Comps.). El jaguar en el nuevo milenio. Fondo de Cultura Económica- Universidad Nacional Autónoma de México-Wildlife Conservation Society. México, D. F.

Ojasti, J. 2000. Manejo de fauna silvestre neotropical. Smithsonian Institution. Washington, D. C.

Ortiz, T. J. 2000. Densidad de población y uso de hábitat del venado cola blanca Odocoileus virginianus en los municipios de Amatlán, Lachatao y Yavesía, Sierra Norte de Oaxaca. Tesis de Maestría. Instituto de Ecología, A. C. Xalapa, Veracruz.

Ortiz-Martínez, T., Gallina, S. \& Briones-Salas, M. 2005. Densidad poblacional y caracterización del hábitat del venado cola blanca (Odocoileus virginianus oaxacensis, Goldman \& Kellog, 1940) en un bosque templado de la Sierra Norte de Oaxaca, México. Acta Zoológica Mexicana (n. s.), 21: 65-78.

Pérez-Mejía, S., Mandujano, S. \& Martínez-Romero, L. E. 2004. Tasa de defecación del venado cola blanca, Odocoileus virginianus mexicanus, en cautividad en Puebla, México. Acta Zoológica Mexicana (n. s.), 20: 167-170.

Piña, E. 2010. Escenarios de manejo sustentable para el venado cola blanca Odocoileus virginianus (Zimmermann, 1780) en la comunidad de Santa María Yavesía, Oaxaca, México. Tesis de maestría. Posgrado en Ciencias Biológicas. UNAM.

Polisar, J. 2002. Componentes de la base de presas de jauguar y puma en Piñero, Venezuela, pp. 151182. In: Medellín, R. A., C. Equihua, C. L. B. Chetkiewicz, P. G. Crawshaw, A. Rabinowitz, K. H. Redford, J. G. Robinson, E. W. Sanderson \& A. B. Taber (Comps.). El jaguar en el nuevo milenio. Fondo de Cultura Económica- Universidad Nacional Autónoma de México-Wildlife Conservation Society. México, D. F. 
Pronatura. 2009. Conservación de tierras. Consultado el $1^{\circ}$ de octubre de 2009: http://www.pronatura. org.mx/tierras_para_conservacion.php

Ramos, F. En proceso. Comunalidad y Resistencia: entre el sacrificio y el gozo en la comunidad de Santa María Yavesía, Sierra Juárez, Oaxaca. Tesis de maestría. Universidad Autónoma Metropolitana-Xochimilco.

Reyna-Hurtado, R. \& Tanner, G. W. 2005. Habitat preferences of ungulates in hunted and non-hunted areas in the Calakmul forest, Campeche, Mexico. Biotropica, 37: 676-685.

Rogers, L. L. 1987. Seasonal changes in defecation rates of free-ranging white-tailed deer. Journal of Wildlife Management, 51: 330-333.

Román, T. 1994. Estimación poblacional del venado cola blanca (Odocoileus virginianus) en bosques montanos de Jalisco y Colima. Tesis de Licenciatura. Facultad de Estudios Superiores Iztacala, UNAM. México.

Rost, G. R. \& Bailey, J. 1979. Distribution of mule deer and elk in relation to roads. Journal of Wildlife Management, 43: 634-641.

Sáenz, J. C. \& Carrillo, E. 2002. Jaguares depredadores de ganado en Costa Rica: ¿Un problema sin solución?, pp. 127-137. In: Medellín, R. A., C. Equihua, C. L. B. Chetkiewicz, P. G. Crawshaw, A. Rabinowitz, K. H. Redford, J. G. Robinson, E. W. Sanderson \& A. B. Taber (Comps.). El jaguar en el nuevo milenio. Fondo de Cultura Económica- Universidad Nacional Autónoma de MéxicoWildlife Conservation Society. México, D. F.

Sánchez-Rojas, G., Aguilar-Miguel, C. \& Hernández-Cid, E. 2009. Estudio poblacional y uso de hábitat por el venado cola blanca (Odocoileus virginianus) en un bosque templado de la Sierra de Pachuca, Hidalgo, México. Tropical Conservation Science, 2: 204-214.

SEMARNAT. 2000. Estrategia nacional para la vida silvestre. Logros y retos para el desarrollo sustentable. 1995-2000. Secretaría de Medio Ambiente y Recursos Naturales-Instituto Nacional de Ecología. México, D. F.

SEMARNAT. 2009a. Sistema de Unidades de Manejo para la Conservación de la Vida Silvestre (SUMA). Consultado el 17 de noviembre de 2009: http://www.semarnat.gob.mx/gestion ambiental/vidasilvestre/Pages/UMA.aspx

SEMARNAT. 2009b. Información de trámites. Vida silvestre. Consultado el 18 de noviembre de 2009: http://www.semarnat.gob.mx/tramitesyservicios/informaciondetramites/Pages/vidasilvestr e.aspx

Smith, W. P. 1991. Odocoileus virginianus. Mammalian Species, The American Society of Mammalogists, 338: 1-13.

StatSoft, Inc. 2004. STATISTICA (Data analysis software system). Versión 7.

Swenson, J. E. 1982. Effects of hunting on habitat use by mule deer on mixed-grass prairie in Montana. Wildlife Society Bulletin, 10: 115-120.

Valenzuela, D. 1991. Estimación de la densidad y distribución de la población de venado cola blanca (Odocoileus virginianus Rafinesque, 1832) en el bosque La Primavera, Jalisco. Tesis de Licenciatura. Universidad Autónoma de Guadalajara.

Villarreal, J. G. 2006. Venado cola blanca: manejo y aprovechamiento cinegético. $2^{\text {a }}$ ed. Unión Ganadera Regional de Nuevo León - Fundación PRODUCE Nuevo León, A. C. - Confederación Nacional de Organizaciones Ganaderas.

Villarreal, J. G. \& Treviño, A. 1999. Estimación de la densidad de población de venado cola blanca "texano" Odocoileus virginianus texanus utilizando el conteo de excretas. Informe de Campo.

Villarreal-Espino, O. A. 2002. El "Grand-Slam" de venado cola blanca mexicano, una alternativa sostenible. Archivos de Zootecnia, 51: 187-193.

Waller, D. M. \& Alverson, W. S. 1997. The white-tailed deer: a keystone herbivore. Wildlife Society Bulletin, 25: 217-226. 
Weber, M., García-Marmolejo, G. \& Reyna-Hurtado, R. 2006. The tragedy of the commons: Wildlife Management Units in Southeastern Mexico. Wildlife Society Bulletin, 34: 1480-1488.

Wilson, D. E., Nichols, J. D., Rudran, R. \& Southwell, C. 1996. Introducción, pp. 1-7. In: Wilson, D. E., F. R. Cole, J. D. Nichols, R. Rudran \& M. S. Foster (Eds.). Measuring and monitoring biological diversity. Standard Methods for Mammals. Smithsonian Institution Press. Washington.

Wong, J. C. 2011. Determinación de peligro a incendios forestales en el municipio de Santa María Yavesía, Oaxaca, México. Tesis de Maestría. Posgrado en Ciencias Biológicas. UNAM.

WWF. 2003. Participan representantes de comunidades oaxaqueñas en el V Congreso Mundial de Parques en Sudáfrica. Boletín de prensa (13 de octubre de 2003). Consultado el 30 de septiembre de 2009: http://www.wwf.org.mx /wwfmex/archivos/bm/031011_participan represent.php

Zar, J. H. 1999. Biostatistical analysis. $4^{\mathrm{a}}$ ed. Prentice Hall. Upper Saddle River, Nueva Jersey. 